\title{
Energetics of macroscopic helical domain in different tube geometries and loading
}

\begin{abstract}
R. H. ZHOU ${ }^{1}$ and Q. P. SUN ${ }^{1 *}$
${ }^{1}$ Department of Mechanical Engineering, The Hong Kong University of Science and Technology, Clear Water Bay, Hong Kong, China

ABSTRACT. Superelastic NiTi polycrystalline shape memory alloy tubes, when subject to slow quasistatic stretching, transform to a high strain phase by the formation and growth of a macroscopic helix-shaped domain as deformation progresses. This paper performed an experimental study on the effects of the external applied nominal strain and the tube geometry (tube radius $R$, wall-thickness $h$ and length $L$ ) on the helical domains in isothermal stretching of the tubes. The evolution of the macroscopic domains with the applied strain in different tube geometries are quantified by in-situ optical measurement. We demonstrate that the equilibrium shape of the macroscopic helical domain and its evolution are governed by the competition between the domain front energy and the elastic-misfit bending strain energy of the tube system. The former favors a short helical domain, while the latter favors a long slim helical domain. The experimental results provided basic physical and experimental foundations for further modelling and quantification of the macroscopic domain morphology evolution in tube geometries.
\end{abstract}

Keywords: NiTi shape memory alloy, martensitic phase transformation, macroscopic helical domains, tube geometry, domain front energy and misfit bending strain energy.

\section{INTRODUCTION}

The basic phenomenon of distinct deformation domain formation and evolution in superelastic NiTi polycrystalline tubes observed in recent of experiments [1-3] is that under displacement-controlled quasi-static uniaxial stretching, phase transition of the polycrystal tube is localized in a helix-shaped martensite (M) domain consisting of almost fully transformed grains with an average strains (elastic strain plus transformation strain) of about $\varepsilon_{y y}=6 \%, \varepsilon_{z z}=-3 \%$ and $\varepsilon_{x x}=-3 \%$ in axial (y), radial (z) and circumferential $(\mathrm{x})$ directions. The domain is separated from the homogeneous elastically deformed austenite (A) matrix (with an axial strain of $\varepsilon_{y y}=1 \%$ ) by the domain front of a finite thickness. Under continued stretching, this high-strain helical domain grows via the propagation of the domain front (a macroscopic interface) before it is eventually merged into a cylindrical domain [3-5].

Generally speaking, domain patterns and their evolutions in different specimen configurations under

* Author to whom correspondence should be addressed; electronic mail: meqpsun@ust.hk, Tel: (852) 23588655, Fax: (852) 23581543.

This is an Open Access article distributed under the terms of the Creative Commons Attribution-Noncommercial License 3.0, which permits unrestricted use, distribution, and reproduction in any noncommercial medium, provided the original work is properly cited. 
external loading are governed by nonlinear interaction of structure (tube geometry) and material properties as represented by different energy terms under the constraint of the tube geometry. For a tube sample containing one helical domain, two energy terms are distinct and their interactions must be emphasized. The first energy term which influences the domain evolution in tube is the bending strain energy caused by the overall elastic misfit in tube circumference between the untransformed (A) and the helical region (Figure 1 (a)). This energy term is sensitive to the domain pattern (whether it is slim or fat), which in turn influences the domain evolution. The second is the macroscopic front energy of the helical domain, arising as the strain misfit along tube wall-thickness direction between A and M phases. It is noticed that there is almost no in-plane mismatch across the domain front in the tube mid-surface since the front always orients at about $55^{\circ}$ to the sample axis (i.e., an in-variant-line as shown by LL' in Figure 1 (b)). Due to the nature of the transformation strain, the domain front is not a habit-plane or invariant-plane as usually found in some single crystals [6, 7] This fact makes the domain front energy density (i.e., the interfacial energy density of the macroscopic domain) scales with the thickness of the tube-wall [8]. More important, such front energy competes with the bending energy of the tube in minimizing the total free energy of the tube system and therefore plays an important role in determining the domain patterns. Therefore, the tube containing one macroscopic helical domain is an ideal configuration to examine the above energetic pictures of domain evolution in different geometries and loading conditions.

In this paper, we aim to develop a simple but well-controlled testing device to perform an in-situ observation of the morphology evolution of the helical domain (characterized by coil number of the helical domain, $N$ ). The variation of domain shape and size with the externally applied stretching (applied nominal strain) in different tube geometries (length $L$, wall-thickness $h$ and radius $R$ ) will be quantified. We focus on the effect of geometric misfit and the associated two energy terms on the domain pattern evolution.

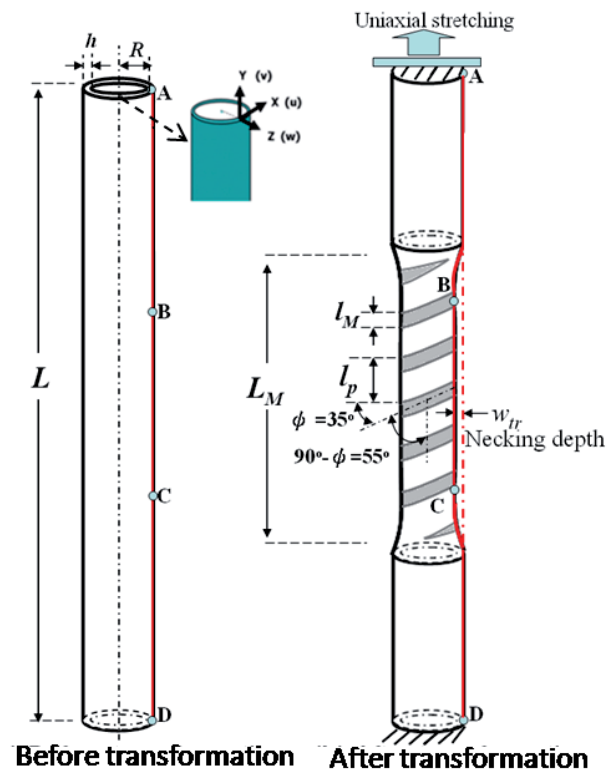

(a)

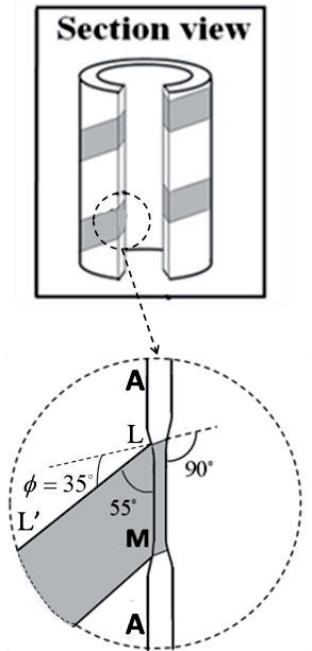

(b)

Fig. 1. (a) Shape of the helical domain in a tube and the overall necking leading to the bending energy of the tube; (b) the macroscopic misfit between martensite and the austenite regions along the wall-thickness direction, which contributes to the main part of the front energy of the macroscopic domain. 


\section{Materials and experimental setup}

The specimens used in the experiment are commercial polycrystalline NiTi tubes (NDC company, USA). A total of 7 specimens were prepared from three different tubes (Tube 1: T1-S1, T1-S2, T1-S3; Tube 2: T2-S1, T2-S2, T2-S3; Tube 3: T3-S1; as listed in Table 1). They were etched into dog-bone shapes by hydrofluoric-nitric acid solution of a volume fraction $\mathrm{HF}: \mathrm{HNO}_{3}: \mathrm{H}_{2} \mathrm{O}=1: 8: 16$ and were used in the test. To facilitate the observation of the domain morphologies on the tube surface, the samples were mechanically polished by fine grained sand papers to reach a final surface roughness of about $0.15 \mu \mathrm{m}$. The austenite finish temperatures $\left(A_{f}\right)$ for all the tubes are below the testing temperature (23 ${ }^{\circ} \mathrm{C}$ ), so the material is in the austenite state and will exhibit superelastic behavior under stress.

Table 1. Geometric dimensions of NiTi tube materials used.

\begin{tabular}{|l|c|c|cc|c|}
\hline \multirow{2}{*}{ geometry } & \multicolumn{3}{|c|}{ Tube 1 } & \multicolumn{3}{c|}{ Tube 2 } & Tube 3 \\
\cline { 2 - 6 } & T1-S1 T1-SS T1-S3 & T2-S1 & T2-S2 T2-S3 & T3-S1 \\
\hline gauge length $L(\mathrm{~mm})$ & 60 & 30 & 60 & 60 \\
\hline wall thickness $h(\mathrm{~mm})$ & 0.10 & 0.16 & 0.10 & 0.04 & 0.10 \\
\hline mean radius $R(\mathrm{~mm})$ & 0.27 & 0.81 & $0.78 \quad 0.75$ & 2.43 \\
\hline
\end{tabular}

The tubes are all tested in a standard mechanical testing machine (MTS, model UTM-RT/10). To minimize the transformation latent heat induced self-heating/cooling effects so as to achieve an isothermal condition for the domain growth/shrink, all tests were performed at a very slow nominal strain rate (=grip-velocity/gauge-length) of $1.0 \times 10^{-5} \mathrm{~s}^{-1}$. To avoid the effect of cyclic loading on the testing results, each test was conducted on a fresh specimen and each specimen was subjected to only one loading-unloading cycle.

The macroscopic helical domain morphologies during the loading were recorded by a CCD camera (JVC TK-C1381) with 25 frames per second (fps). One circular lamp to light the two side edges of the curved surface and one straight lamp to reflect the middle portion of the tube were used to illuminate the whole curved surface of observation for the small diameter tubes. As shown in Figure 1, the length of the helical domain is characterized by the coil number $N$ of the helix defined as:

$$
N=L_{M} / l_{p},
$$

with $L_{\mathrm{M}}$ the axial distance between the two helix tips, and $l_{\mathrm{p}}$ the pitch length of the helix domain.

In experiments, $N$ was determined by the two helix tips from the measured domain patterns. The variation of helical domain morphology in the loading process was synchronized with the measured nominal stress-strain curves by the developed software [3]. In order to observe the whole processes of the helical domain growth and shrinkage in loading and unloading, all the tube samples were unloaded just before the helical domain is merged into a cylindrical domain.

The geometric misfits in radial and wall-thickness directions due to the presence of the helical domain were measured by the optical profiler (Model Wyco NT3300, VEECO) so as to demonstrate the bending strain energy and domain front energy.

\section{Experimental Results and discussion}

\subsection{Bending strain energy due to radial misfit}

The presence of a helical domain (with transformation strain $\varepsilon_{x x}^{t r}=5 \%$ in the axial direction, $\varepsilon_{x x}^{t r}=-2.5 \%$ 
in circumferential direction and $\varepsilon_{z z}^{t r}=-2.5 \%$ in wall-thickness direction) causes a overall reduction in tube circumference and local reduction in tube wall-thickness as schematically shown in Figure 1 (a), (b) respectively. The reduction in the circumference leads to an overall inward radial necking which misfits with the untransformed section of the tube. The necking profiles of tube generator at different stages of helical growth were measured by optical profiler as shown in Figure 2 (a). The bending strain energy due to the necking misfit is mainly stored in the two transition regions with characteristic length $l_{b}$ around the joints (as shown in Figure 2 (a)). By geometric analysis, the overall necking depth $w_{t r}$ due to the presence of the helical domain is

$$
w_{t r}=R \cdot \varepsilon_{x x}^{t r} \cdot S F=R \cdot S F \cdot\left(\varepsilon_{y y}^{t r} / 2\right)
$$

where $S F=l_{M} / l_{p}$ is the slimness factor of the helix, $l_{M}=\left(L \cdot \varepsilon_{*}\right) /\left(N \cdot \varepsilon_{y y}^{t r}\right)$ is the width of each helical coil; $\varepsilon_{*}$ is the nominal tensile transformation strain. As shown in Figure 2 (b), the measured value of $w_{t r}$ was found to increase linearly with $S F$ of the helix, agreeing well with Eq. (2). The bending energy will be calculated later.

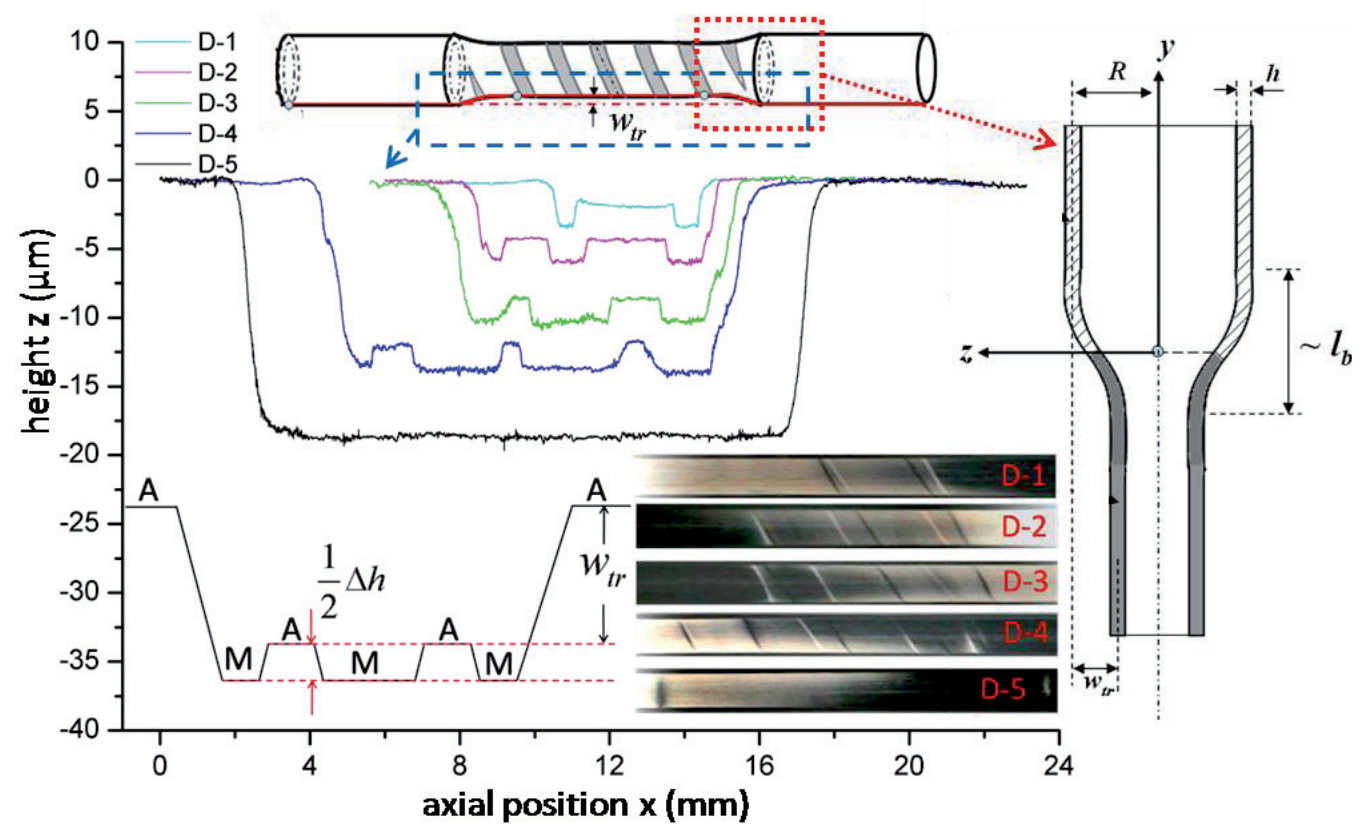

(a) 


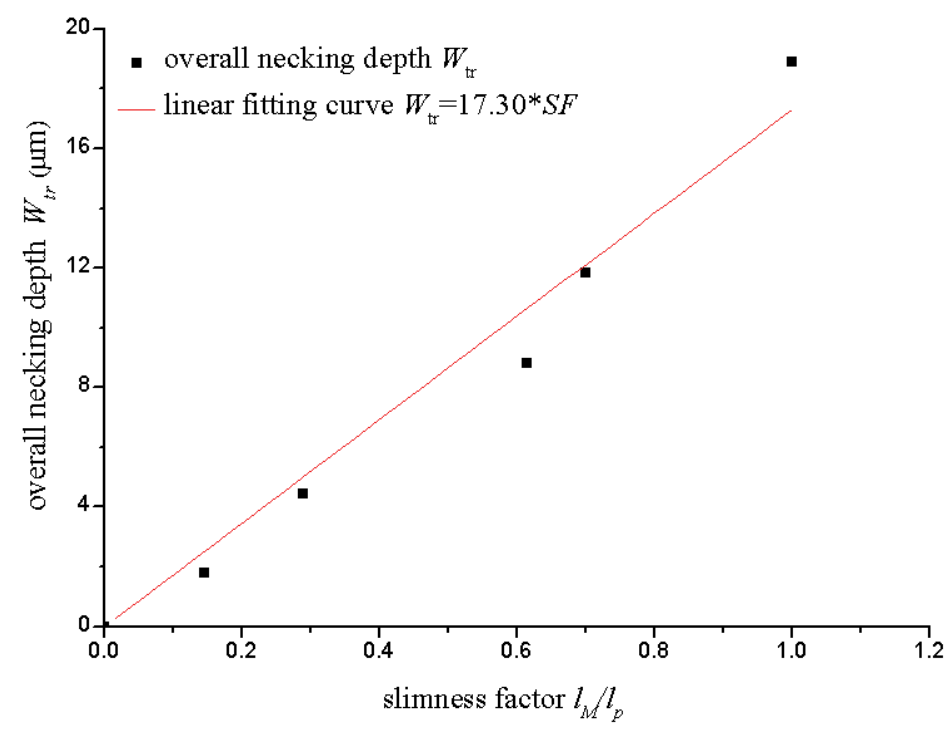

(b)

Fig. 2. (a) 5 surface profiles measured for the calculation of the tube mid-surface deflection; (b) the linear dependence of the measured necking depth on domain patterns (i.e. $S F$ ).

\subsection{Domain front energy due to the tube wall-thickness misfit}

The reduction of tube-wall thickness in the martensite domain causes a misfit between $\mathrm{A}$ and $\mathrm{M}$ along thickness ( $\mathrm{z}$ ) direction. The misfit creates macroscopic misfit stress and domain front energy which is proportional to the interface (front) area. We used thin plate specimens (Figure 3 (a)) under tension to determine the frontal region (front thickness $l_{c}$ ), local thickness reduction $(\Delta h)$ and the dependence of the domain front energy density on the plate thickness $(h)$. From the measured axial front thickness $l_{a}=l_{c} / \cos \varphi$ with $\varphi$ the orientation angle $\left(\varphi=35^{\circ}\right.$ as shown in Figure $\left.3(\mathrm{a})\right)$, it was found that both $l_{a}(\approx h$, so $\left.l_{c}=h \cos \varphi\right)$ and $\Delta h(=2.54 \% h)$ are proportional to the wall thickness $h$, as indicated in Figure 3 . We can estimate the front energy density $\gamma_{\text {front }}$ as

$$
\gamma_{\text {front }}=\lambda \cdot l_{c} \cdot E \cdot\left(\varepsilon_{z z}^{t r}\right)^{2}
$$

where $\lambda$ a coefficient only dependent on Poisson's ratio $v$ of the material.

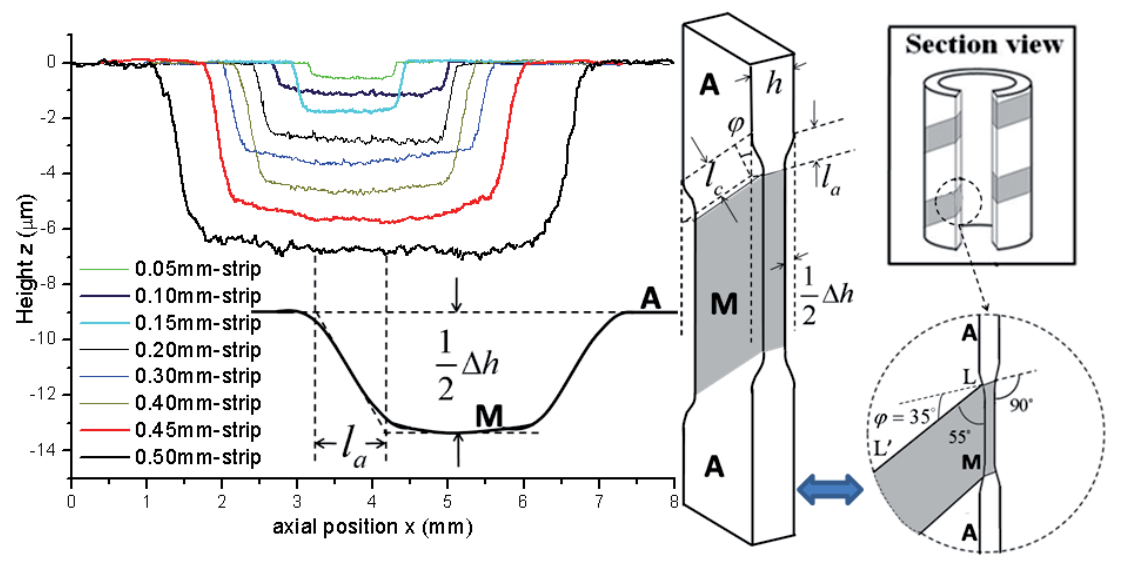

(a) 


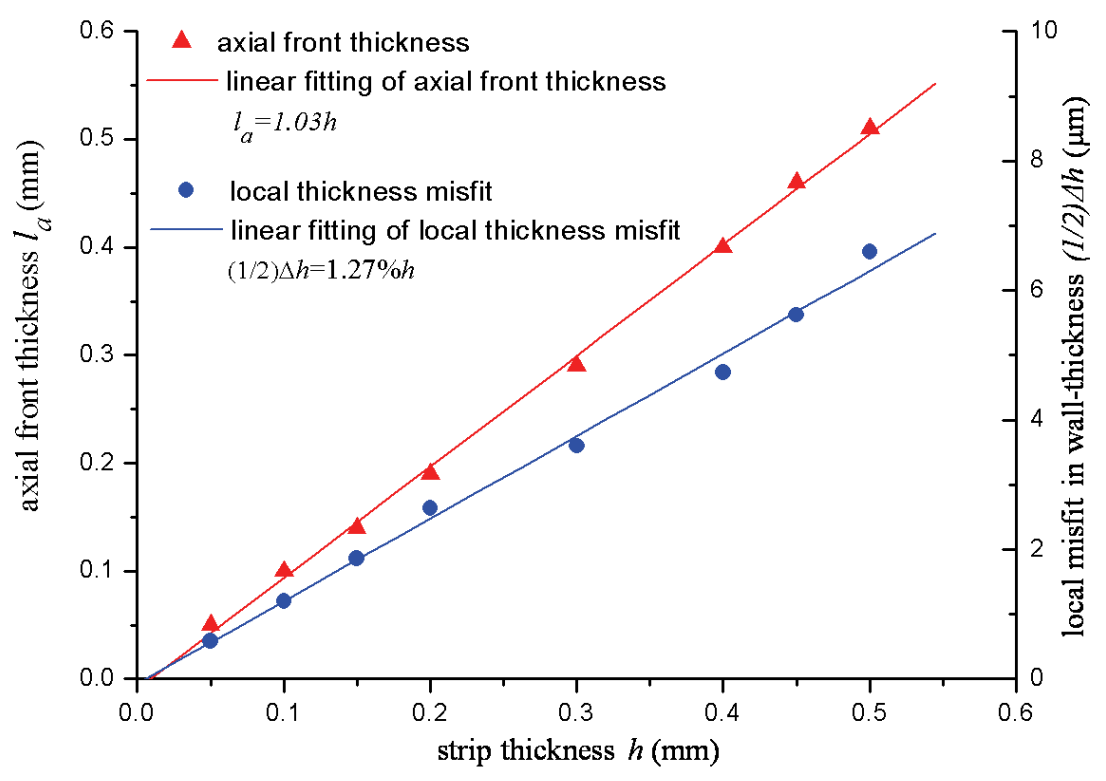

(b)

Fig. 3. (a) Surface profiles domains in of strips of different thicknesses; (b) the dependence of the local thickness misfit $(\Delta h)$ and axial front thickness $l_{\mathrm{a}}$ on strip thicknesses $h$.

\subsection{Energy minimization and scaling law of equilibrium helical domain}

According to Ref. [9], the bending strain energy $U_{b e n d}$ of the tube can be expressed as

$$
U_{\text {bend }}=2 \pi R \cdot h \cdot l_{b} \cdot E \cdot\left(\frac{w_{t r}}{2 R}\right)^{2}=A_{1} \cdot \frac{1}{N^{2}} \cdot l_{b} \cdot \frac{h}{R} \cdot\left(L \cdot \varepsilon_{*}\right)^{2}
$$

where $A_{1}=\frac{E}{32 \pi \cdot \tan ^{2} \phi}, \quad l_{b}=\left(\frac{1}{3\left(1-v^{2}\right)}\right)^{\frac{1}{4}} \sqrt{R \cdot h}$ is the characteristic length of the necking transition zone. The total domain front energy $\Gamma$ of the helix can be expressed as

$$
\Gamma=N \cdot \frac{4 \pi \cdot R \cdot h \cdot \gamma_{\text {front }}}{\cos \phi}
$$

It is seen that for a given $\varepsilon_{*}$ the bending strain energy inversely scales with $N$ by an exponent 2 , while domain front energy linearly scales with $N$. From energy point of view, the bending strain energy favors a long (large $N$ ) helical domain, while the domain front energy favors a short ( small $N$ ) helical domain.

We can apply the energy minimization principle to determine the equilibrium coil number $N$ of the helical domain. It is shown that the $N$ depends on the tube geometry $(L, h, R)$ and nominal transformation strain $\mathcal{E}_{*}$ by the following scaling law [9],

$$
\frac{N}{C_{1}}=\left(\frac{1}{\lambda}\right)^{1 / 3} \cdot\left(\varepsilon_{*}\right)^{2 / 3}, \text { with } C_{1}=\left[\left(\frac{1}{3\left(1-v^{2}\right)}\right)^{1 / 4} \cdot \frac{\cos \phi}{16 \cdot\left(\pi \cdot \operatorname{tg} \phi \cdot \varepsilon_{y y}^{t r}\right)^{2}} \cdot \sqrt{\frac{R}{h}} \cdot\left(\frac{L}{R}\right)^{2}\right]^{1 / 3} .
$$

This scaling law relationship can also be re-arranged to, 


$$
N=K_{t h} \cdot\left(\frac{R}{h}\right)^{\frac{1}{6}} \cdot\left(\frac{L \varepsilon_{*}}{R \varepsilon_{y y}^{t r}}\right)^{\frac{2}{3}}
$$

where $K_{t h}=\left(\frac{1}{\lambda}\right)^{1 / 3} \cdot\left[\frac{1}{3\left(1-v^{2}\right)}\right]^{1 / 12} \cdot \frac{\cos \phi}{16 \cdot(\pi \cdot \operatorname{tg} \phi)^{2}}$.

The experimental results of the length of the helical domain $N$ in the 7 tube samples used in this work are shown in Figure 4. It is seen that $N$ depends on the product of two dimensionless quantities $\left(\frac{R}{h}\right)^{\frac{1}{6}} \cdot\left(\frac{L \varepsilon_{*}}{R \varepsilon_{y y}^{t r}}\right)^{\frac{2}{3}}$ in a linear manner, which agrees well with Eq. (7) (as shown in Figure 4, $K_{t h}$ $=0.95)$.

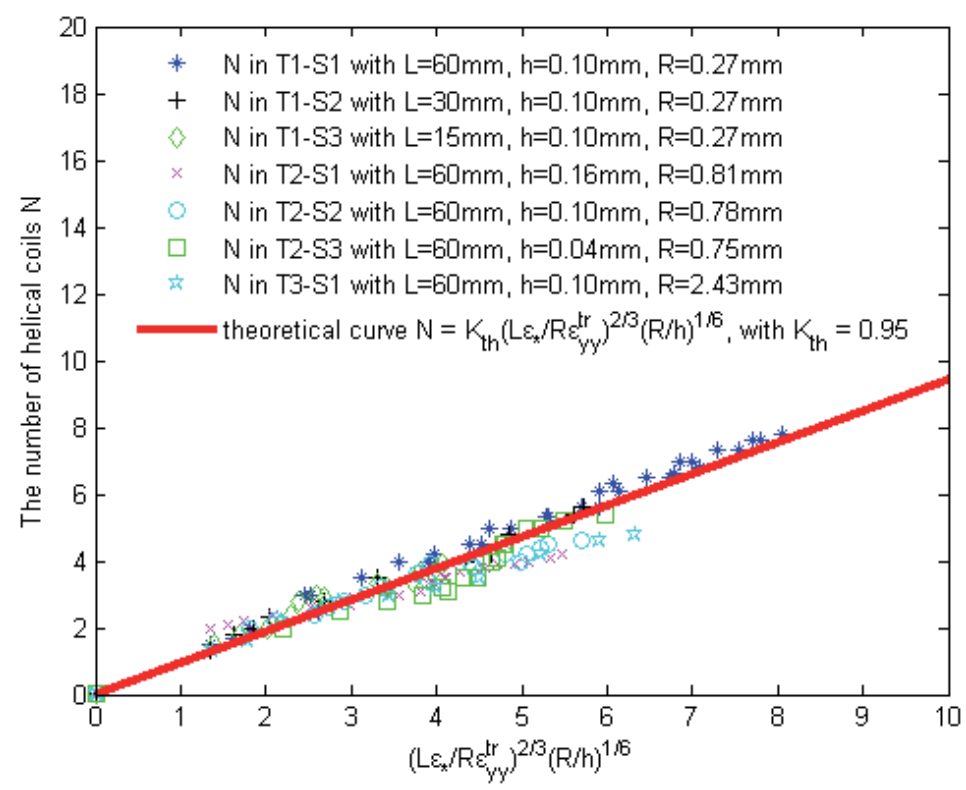

Fig. 4. The experimental measurements of the coil number $N$ of the helical domain in seven different NiTi tube samples used and the theoretical prediction Eq. (7).

\section{Conclusions}

In this paper a simple and well-controlled testing device for an in-situ observation of the morphology evolution of the helical domain (characterized by the number of helical coils, $N$ ) was developed. The variation of domain shape and size with the externally applied stretching (applied nominal strain) in different tube geometries (length $L$, wall-thickness $h$ and radius $R$ ) were measured. We focus on the main energy terms in the domain evolution during phase transformation of the tube. Through experiment, we demonstrated and quantified the two important energy terms in understanding the observed various helical domain patterns in different sized NiTi shape memory alloy tubes under tension: bending strain energy and domain front energy. It is shown that the two energy terms depend on external applied nominal strain and the tube geometry (tube radius $R$, wall-thickness $h$ and length $L$ ). 
From thermodynamic point of view, the bending strain energy favors a long helical domain while the domain front energy favors a short helical domain. These two energy terms compete in minimizing the tube's energy and therefore plays an important role in the equilibrium helical domain pattern. The experimental measurement of helical domain shapes agree well with the scaling law derived from theoretical thermodynamic analysis on the equilibrium domain patterns.

\section{Acknowledgement}

The authors are grateful to the financial support from the Research Grants Council of the Hong Kong

SAR, China through Project No. 619806.

\section{References}

1. Li, Z.Q., Sun, Q.P.. The initiation and growth of macroscopic martensite band in nano-grained NiTi microtubes under Tension. Int. J. Plasticity 18, 1481-1498 (2002)

2. Sun, Q.P., Li, Z.Q.. Phase transformation in superelastic NiTi poly- crystalline micro-tubes under tension and torsion-from localization to homo- geneous deformation. Int. J. Solids Struct. 39, 3797-3809 (2002)

3. Feng, P., Sun, Q.P.. Experimental investigation on macroscopic domain formation and evolution in polycrystalline NiTi microtubing under mechanical force. J. Mech. Phys. Solids 54, 1568-1603 (2006)

4. Li, Z.Q., Sun, Q.P.. Superelastic NiTi memory alloy micro-tube under tension-nucleation and propagation of martensite band. Key Eng. Mater. 177-180, 461-466 (2000)

5. Ng, K.L., Sun, Q.P.. Stress-induced phase transformation and detwinning in NiTi tubes. Mech. Mater. 38, 41-56 (2006)

6. Wayman, C.M.. Introduction to Crystallography of Martensitic Transformations. Macmillan, New York (1964)

7. Roitburd, A.L.. Domain structure of crystals formed in a solid phase. Soviet Physics - Solid State 10, 2870 (1968)

8. Cahn, J.W., Hilliard, J.E.. Free energy of a nonuniform system I. Interfacial energy. J. Chem. Phys. 28 (2), 258-267 (1958)

9. He, Y.J., Sun, Q.P., 2009. Scaling Relationship on macroscopic helical domains in NiTi tubes. Int. J. Solids Struct. 46, 4242-4251 (2009) 